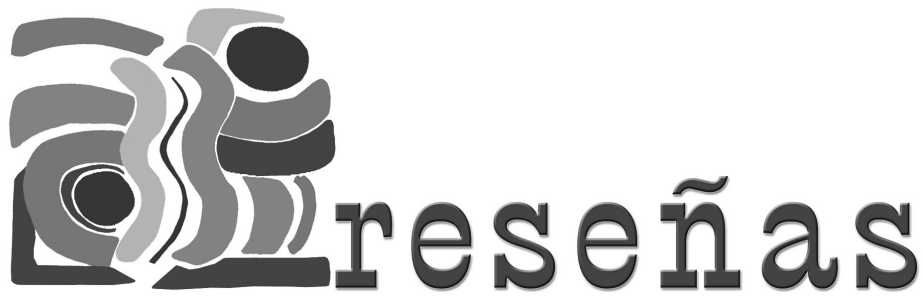

\title{
Acción de tutela en salud: piedra en el zapato en la consolidación de las reformas de mercado
}

Echeverry ME, compiladora. Indignación justa: Estudios sobre la Acción de Tutela en Salud en Medellín. Medellín: Facultad Nacional de Salud Pública - Universidad de

Antioquia; 2013*

\section{Por: Mauricio Torres-Tovar}

doi: 10.11144/Javeriana.rgyps13-27.atsp

Cómo citar esta reseña:

Torres-Tovar M. Acción de tutela en salud: piedra en el zapato en la consolidación de las reformas de mercado, reseña de Echeverry ME, compiladora. Indignación justa: Estudios sobre la Acción de Tutela en Salud en Medellín. Medellín: Facultad Nacional de Salud Pública - Universidad de Antioquia; 2013. Rev. Gerenc. Polít. Salud. 2014; 13(27): 363-369. http://dx.doi.org/10.11144/ Javeriana.rgyps13-27.atsp

[...] para proteger los derechos humanos no basta solo con proclamarlos, se trata de saber cuál es el modo más seguro de garantizarlos, para impedir que a pesar de las declaraciones solemnes sean continuamente violados.

Norberto Bobbio

El libro, editado y compilado por la profesora Esperanza Echeverry López, de la Facultad Nacional de Salud Pública de la Universidad de Antioquia, titulado Indignación justa: Estudios sobre la Acción de Tutela en Salud en Medellín, sin lugar a dudas se constituye en un referente académico y político a la hora de estudiar e interpretar las acciones de exigibilidad jurídica del derecho a la salud en Colombia.

La obra compila cuatro investigaciones realizadas por profesores y estudiantes de la Facultad Nacional de Salud Pública sobre varios aspectos de la acción de tutela en salud (ATS) realizadas en Medellín durante el periodo 2001-2009. Estas investigaciones, a diferencia de otras que se han realizado en el país, tienen como novedad el hecho que sitúan el análisis de las ATs en el campo sociosanitario y en el de los costos del proceso para el sistema judicial y para la sociedad.

Estos análisis se establecen como indicadores para el conocimiento del acceso a los servicios de salud, ${ }^{1}$ del sufrimiento personal y familiar que

* Presentación realizada en la ciudad de Medellín, en la Biblioteca Pública Piloto, el día 19 de agosto de 2014.

1. En tanto el indicador que se viene usando exclusivamente es el de cobertura de aseguramiento y se sabe que aseguramiento no es igual a acceso.
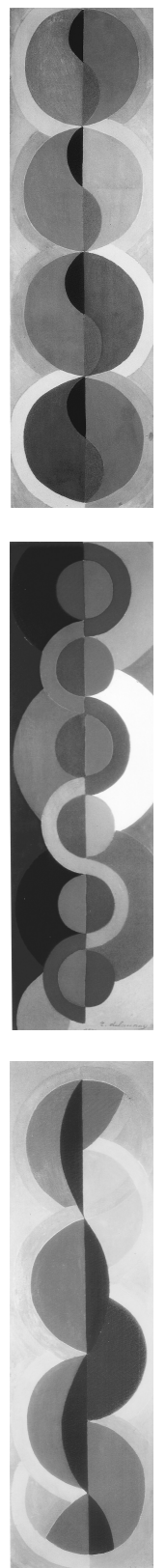
producen las formas como los ciudadanos tienen que relacionarse con el sistema de salud, del deterioro de la situación de salud que produce este tipo de sistema, y contribuye con más evidencias acerca de los límites estructurales para la garantía del derecho a la salud de los arreglos institucionales centrados en el mercado que se establecieron con la reforma en salud del año 1993 en Colombia.

El libro presenta aristas del tema de la ATS que otras investigaciones no habían tocado, como es la de los itinerarios detallados que se dan en el proceso de uso de la ATs, las razones para su uso y no uso, y sobre los costos que acarrea al sistema judicial, para contrastar con aquellos que han centrado su atención en los costos que las ATs derivan para el sistema de salud.

\section{Lo que revela el uso de la ATs}

El libro, a partir de los hallazgos de las investigaciones, revela una gran cantidad de hechos que se desprenden del análisis del uso de la ATs.

Tal vez, el hecho más relevante es que el uso de las ATs es el reflejo de la violación sistemática y persistente del derecho a la salud (DS) en Colombia, situación que debe ser entendida y analizada desde el drama, el sufrimiento y la humillación que sufren los pacientes y sus familias, producto de lo que significa la negación de servicios de salud cuando se requieren y en el itinerario que se debe recorrer al emprender el uso de la ATS.

A la vez, revela la indignación y la resistencia que llevan a que los y las ciudadanas emprendan este tipo de acciones para exigir la garantía de su derecho a la salud, con lo que se evidencian las demandas ciudadanas en salud, que no son otra cosa que la expresión de sus necesidades encarnadas en el derecho a la salud.

Igualmente, el uso amplio de la ATS expresa el profundo descredito e ilegitimidad social que tiene el sistema de salud en Colombia, razón por la cual como lo menciona el libro, el uso de las ATs hay que verlo en conjunto con las expresiones colectivas de movilizaciones y protestas sociales y con las propuestas alternativas derivadas de los sectores sociales para transformar el sistema de salud. Aunque algunas acciones individuales han venido ganando espacio como repertorios de acción colectiva que tienen facetas individuales, pero también grupales.

Las ATS han permitido acceso a los servicios de salud y han establecido un horizonte ético-político para la garantía del Ds, pero en un contexto inequitativo, en donde su uso se hace de manera dispar y donde lo sectores con más necesidades en salud son los que menos acceden a ella; igualmente, hay que reconocer que existe una baja eficacia de la ATs para la garantía del derecho, debido principalmente a que las EPS e IPS acatan solo parcialmente los mandatos de los jueces y a que el uso de los recursos posteriores a la tutela, es poco común (como es el caso de la acción de desacato), así como también un mecanismo muy débil por parte del sistema judicial para garantizar que sus mandatos se cumplan.

Las investigaciones dejan en claro que el no cumplimiento de la ATs genera consecuencias tanto sanitarias como económicas. Es decir, el que no se atienda a la gente a su debido tiempo, deteriora la condición de salud para muchos, asunto totalmente evitable, y que el retraso de la atención suele encarecerla dado que los procesos asistenciales requieren mayor complejidad tecnológica. Y como se sabe, suele dejar deterioro permanente para muchos y para otros el costo ha sido su propia vida. 
De manera clara, el libro deja explicito que la ATS no afecta lo estructural del Sistema General de Seguridad Social en Salud (sGSss), pero tal como lo dice metafóricamente, se constituye en una piedra en el zapato en la consolidación de la reforma de mercado.

Desde el análisis político que se realiza en el libro, se revela la tensión que ha existido entre el enfoque del Estado Social de Derecho (ESD) y la tradición liberal clásica, es decir, entre el enfoque de derechos sociales y el del mercado, que tienen visiones opuestas frente a las estrategias para garantizar las necesidades de salud de la población y que han establecido una clara contienda política por el control de la salud en Colombia entre los sectores proclives al derecho a la salud y los sectores proclives al mercado.

Sin lugar a dudas, el libro revela el papel trascendental que han tenido los jueces y la Corte Constitucional para ir construyendo en la realidad el ESD, para lo cual han generado jurisprudencia en relación con el Ds, teniendo como apoyos el derecho internacional del derecho a la salud y la idea del bloque de constitucionalidad, que permitió llegar a declarar a través de la Sentencia T-760 de 2008 la salud como derecho fundamental. En este sentido, el libro establece que la ATs ha dado lugar a una jurisprudencia no siempre consistente, pero que ha instaurado y ampliado la noción del DS.

Esto, a su vez, ha hecho que la gente crea en la ATs, la utilice y ha llevado a la creación de una cultura incipiente de exigibilidad del derecho a la salud. Pero igualmente, ha generado una situación de amenaza contra la ATs, así como contra la Corte Constitucional.

Las investigaciones también revelan, de manera inédita, por qué la gente no usa la ATS y destaca el tema del miedo, entronizado en el país y destructor de tejido social y de procesos organizativos.

También de manera inédita, el libro revela los costos que acarrea para el sistema judicial las ATS, evidenciando que en un gran porcentaje cuestan más de lo que cuestan los servicios de salud demandados por el ciudadano. Esta arista no ha sido ni tratada, ni tocada por los detractores de la ATs. La sociedad tendría que exigirle a las EPS que paguen los costos que genera su postura de lucro, la cual lleva a la ciudadanía a interponer las ATS.

La obra en su análisis deja claro que el orden institucional y legal en salud en Colombia en la práctica ha desvirtuado los desarrollos jurisprudenciales internacionales del DS, a pesar de las posturas y decisiones tomadas por los jueces y la Corte Constitucional, al configurarlo como un derecho social de prestación que se debe resolver principalmente en el mercado.

El caso colombiano es un buen ejemplo para mostrar que, efectivamente, el campo de la seguridad social es un campo de acumulación de capital, en donde los agentes del mercado han establecido formas rentables, tanto legales como ilegales. Para esto, la noción de DS la configuraron en torno a la capacidad de pago (vía cotización) y al aseguramiento (de un paquete de servicios establecidos), convirtiéndolo en la práctica en un derecho contractual al cual se accede según capacidad de pago y se obtiene lo establecido en las cláusulas contractuales. ${ }^{2}$

Los mecanismos de mercado no funcionan. Las EPS no se comportan como aseguradoras,

2 En este sentido, se considera que el debate entre perspectivas del derecho se da más entre los fundamentales y contractuales, que entre los fundamentales y prestacionales (como se plantea en el libro), dado que los derechos fundamentales tienen contenidos prestacionales. 
hay fracaso de los contratos, la separación de funciones no se da, los mecanismos de regulación estatal tampoco. En este sentido, el libro revela un fracaso del modelo de salud tanto desde la perspectiva de la economía neoclásica, como desde la perspectiva sociosanitaria.

Se evidencia que, realmente, en el sistema de salud colombiano no existe regulación, a pesar de ser una tesis central de la orientación de la reforma de salud, bajo el esquema de un pluralismo estructurado regulado. Y esto en gran medida porque la acción gubernamental ha sido proclive a los intereses del mercado y ha fortalecido la posición dominante de las EPS. En este sentido, se podría decir que el Estado ha sido capturado por los intereses de las EPS.

Es clara la posición dominante de las EPS, que se expresa en la imposición de cláusulas contractuales, discriminación a IPS, estrategias de selección de riesgo, barreras de acceso e itinerarios burocráticos. Se han venido estableciendo como oligopolios y en algunas regiones como monopolios, fortaleciendo su poder de mercado. Hacen una maximización de la rentabilidad económica, con mecanismos de contención de costos y máxima productividad. Han llevado a reconfigurar lo público a favor del mercado y de su posición dominante.

Otro aspecto muy importante evidenciado en el análisis del libro, es que se han establecido estrategias para reconfigurar las maneras de captar las rentas, por lo cual las EPs han hecho adaptación según las medidas de control que han surgido, como en el caso de la Sentencia T-760, donde empezaron a utilizar los recobros para mejorar su lucro.

El otro actor beneficiado con este modelo del sGss -en el decir de los autores del libro- ha sido la industria farmacéutica, que ha obtenido superlucros.
Finamente, se puede decir que como hechos que revela el libro a través de sus investigaciones, se ratifica que el sGsss y sus reformas, no han garantizado el Ds en Colombia, razón por la cual en el país continuamos sin una experiencia histórica de la garantía del derecho a la salud.

\section{Las falacias que han querido imponer}

El análisis realizado por los autores evidencia cómo a la sociedad se le han impuesto debates e ideas que no son necesariamente veraces.

El libro llama la atención sobre las falacias de la escasez de recursos en el sistema de salud, el supuesto abuso con el uso de la tutela y la posibilidad de quiebre financiero que podría generarle ello al sistema de salud.

Desde la perspectiva neoclásica de la economía de la salud, se ha impuesto que ante recursos escasos no es posible garantizar todo lo correspondiente al derecho a la salud. Existen suficientes evidencias investigativas que soportan que uno de los efectos que produjo la reforma de salud en Colombia fue una inyección muy amplia de recursos; en ese sentido, no es cierto que estemos ante un escenario de recursos escasos para la salud en el país. Lo que se debe cuestionar es para qué se han utilizado estos recursos y en manos de quiénes han venido quedando.

Las investigaciones contenidas en el libro muestran claramente que la gente no abusa de la ATs, dado que recurre previamente a otros recursos, incluido el gasto de bolsillo, y que alrededor de un $60 \%$ de lo que demandan las ATs está financiado, dado que hace parte del pos.

En este sentido, es claro que los agentes de mercado en el sGss han buscado ideológicamente favorecer su posición de poder y hacer 
creer que el uso de tutela es inadecuado para la garantía del Ds. Han buscado ubicar como uno de los problemas centrales para el modelo de salud el uso de la ATs y encubrir los reales problemas estructurales derivados del modelo de intermediación y la lógica de lucro impuesta por los agentes de mercado al sistema de salud.

\section{El contexto internacional de la justiciabilidad del derecho a la salud}

En el mundo, según la investigación realizada por Gloppen y Yamin, se evidencia que los juicios relacionados con el Ds se encuentran en aumento. Colombia es uno de los países que sobresale por el volumen de ATs interpuestas por la ciudadanía, como lo revelan las investigaciones de la Defensoría del Pueblo y las contenidas en el libro al cual hacemos referencia en esta presentación.

De esta situación surge un conjunto de preguntas relacionadas con si el uso de mecanismos de justiciabilidad del Ds es positivo o negativo para la promoción de este derecho, si hace la atención más justa, o si profundiza las desigualdades en tanto que quienes acceden tienen mayores capacidades económicas y solicitan servicios exagerados.

Un primer aspecto que es necesario referir acá, es que sin justiciabilidad (que es la exigibilidad jurídica) no es posible la configuración de los derechos, quedarían solo en proclamas como menciona Bobbio. Y como plantea el análisis del libro, incluso se hace necesario ir más allá para su concreción, a través de la movilización jurídica, la justicia distributiva y la justicia sanitaria.

Las investigaciones realizadas en Medellín sobre el uso de ATs dejan en claro que su uso es enteramente positivo para la promoción del Ds y que no buscan una garantía absoluta o ilimitada del contenido del Ds, dado que se refieren principalmente a un contenido específico de la atención en salud y lo que reclaman de manera justa es la integralidad de la atención.

Por lo tanto, la tesis que desde el escenario nacional e internacional se busca imponer a la justiciabilidad del Ds por buscar "el todo", para el contexto colombiano no aplica y no puede aceptarse como argumento para colocar en tela de juicio el uso de la ATs.

Ahora bien, esto no quiere decir que no se hayan dado casos donde los actores de poder como las EPS y la industria farmacéutica hayan buscado aprovecharse de este recurso para su lucro, pero esto no es lo generalizable, y solo debe ser tenido en cuenta para denunciarlo e impedirlo.

Igualmente, en este contexto, el libro se refiere a los escasos avances de la justiciabilidad en el derecho internacional, que aún no tiene los mecanismos e instancias internacionales que sean vinculantes para exigirle a los Estados la garantía del Ds a los colectivos. El protocolo facultativo del Pacto Internacional de Derechos Económicos, Sociales y Culturales (Pidesc) aún está por mostrar sus bondades.

En este contexto internacional, no se puede dejar de mencionar dos asuntos claves en este debate. De un lado, la captura y distorsión de conceptos claves como son la universalidad, el Ds y la equidad. La universalidad es un paquete de aseguramiento para todos, el contenido del derecho es este paquete restricto de servicios de atención, en tanto que la equidad se equipara a subsidios condicionados para los más pobres. Sin lugar a dudas, la batalla de ideas sigue estando al orden del día, para no perder la esencia de categorías fundamentales en la comprensión del DS. 
De otro lado, se hace necesario mencionar que el Banco Mundial desde el año 2010 ha puesto en desarrollo la iniciativa Salud Derecho que busca "generar un espacio de diálogo para compartir conocimiento, desarrollar estrategias y discutir alternativas de solución en temas de equidad, eficiencia, diseño e implementación de políticas públicas sobre priorización, derecho y judicialización de la salud y motivar el análisis sobre la importancia de implementar procesos de priorización en salud que de manera participativa, transparente y legítima, definan los contenidos de los paquetes de beneficios en cada país, y por esta vía, hacer de la priorización un ejercicio a través del cual se accede al derecho a la salud de forma incluyente". Proyecto que claramente busca cooptar y subordinar los procesos de justiciabilidad del derecho a la salud en la región, con la idea de priorización y de paquetes de servicios, que es una forma de limitar el contenido esencial del DS.

\section{El nuevo contexto con la ley estatutaria de salud y la regla fiscal}

La exequible dada por la Corte Constitucional a la Ley Estatutaria en Salud, es un retroceso al reducir el derecho a la salud a un paquete de servicios (POS), en contra de la perspectiva integral que establecen los pactos y tratados internacionales de derechos humanos, e incluso en contra de la perspectiva progresista abierta por la propia Corte Constitucional con la Sentencia T-760 en el 2008, que declaró la salud como un derecho humano fundamental.

Aunque el objetivo de una ley estatutaria en salud es regular este derecho humano, es claro que su garantía por parte de los Estados se concreta según la orientación y estructuración de los sistemas y políticas de salud. Esta ley no modifica en absoluto la matriz de aseguramiento para la atención de la enfermedad, ni la intermediación efectuada por las EPS.

De igual manera, la Ley establece no prestar servicios de salud en los casos que tengan como finalidad principal un propósito cosmético, no exista evidencia científica sobre su seguridad y efectividad clínica, que su uso no haya sido autorizado por la autoridad competente, o no se encuentre en fase de experimentación, o no se preste en el exterior. Con estos criterios se excluyen muchas de las atenciones que la gente necesita para resolver sus problemas de salud.

Por lo tanto, un efecto grave con la Ley Estatutaria es que se da paso a un uso restrictivo de la tutela, en tanto esta protege el contenido jurídico que se le establece al derecho a la salud, que podrá ahora ser interpretado por los jueces solo estrictamente como el contenido del pos, el cual no es suficiente para cubrir necesidades de atención para la gente.

A esto se suma la regla fiscal que se establece como un supraderecho, con la cual no será posible colocar en operación medidas como las ordenadas por la Corte Constitucional a través de la Sentencia T-760 dado que si coloca en riesgo fiscal al país, no es posible acogerlas. Con esto se impone la lógica económica sobre la lógica de la protección de los derechos humanos.

\section{El aporte de este tipo de estudios al campo de la salud pública}

La obra en mención revela una nueva vertiente de interés e investigación en el campo de la salud pública, novedoso para Colombia, que busca interpretar la acción de los sectores subalternos de la sociedad, sus formas de resistencia y acción para la defensa y garantía de su derecho a la salud. 
La ATs es una expresión de la resistencia ciudadana y de la indignación que lleva a la acción. La ATs configura sujetos políticos titulares del Ds, gesta una ciudadanía social y una ciudadanía política, razón por la cual su estudio debe ser parte de las investigaciones que buscan interpretar cómo los individuos y los colectivos se organizan para demandar necesidades y construir identidades.

El libro deja claro que el uso de la ATs tiene una acción restrictiva, en tanto que acción individual, y que se trata de ver cómo permite gestar acciones colectivas y pasar incluso a acciones de justiciabilidad y exigibilidad del Ds colectivas, asunto que ni siquiera está previsto en el orden internacional del DS.

De otro lado, se requiere profundizar por qué a pesar de un estado de violación sistemática del Ds en Colombia, no se dan procesos de indignación generalizados que lleven a movilizaciones sostenidas y a cambios estructurales de un sistema de salud completamente deslegitimado.

\section{Los retos que deja planteados el libro}

El libro deja planteado un conjunto de retos en el ámbito académico y político.

Con estas investigaciones se abren nuevas preguntas y temas de interés para seguir profundizando, como por ejemplo conocer cuáles son los costos económicos con los que acarrean las familias al interponer una ATS y cuál ha sido la incidencia de la ATs en el diseño de política pública en salud.

En lo político, partiendo del reconocimiento de que Colombia no ha vivido una experiencia histórica de ciudadanía social y de garantía del Ds, se requiere para que el derecho no sea solo una proclama, mecanismos efectivos de exigibilidad y control social, una fuerte rectoría del Estado para que sancione y una realización cabal de este a través de la ejecución de políticas públicas.

Pero avanzar en esta vía en Colombia requiere un nuevo pacto social, con una real justicia sanitaria articulada al interés colectivo, y no de mercado, y una redistribución económica, porque sin esta no es posible la garantía de los derechos sociales.

Ideológicamente, se requiere reconocer que no son conciliables el lucro y la garantía del DS, por lo que se requiere transformar la racionalidad mercantil en la salud, lo que implica avanzar en un real reconocimiento de la salud como derecho humano que la desliga de las ideas de caridad, de favor o de capacidad de pago para acceder a ella.

En el contexto de salud actual en Colombia, con la Ley Estatutaria de Salud declarada exequible y una legislación en torno a la regla fiscal, se advierte que en el periodo por venir solo se podrá pensar en algo distinto si se profundiza la movilización social, que sumada a los diálogos con la insurgencia permita generar un nuevo pacto social en Colombia, que dé la posibilidad de establecer una real política y un sistema de salud garante de este derecho para toda la población. 